\title{
Higher randomness and forcing with closed sets
}

\author{
Benoit Monin \\ Université Paris Diderot, LIAFA, Paris, France \\ benoit.monin@computability.fr
}

\begin{abstract}
Kechris showed in [8] that there exists a largest $\Pi_{1}^{1}$ set of measure 0. An explicit construction of this largest $\Pi_{1}^{1}$ nullset has later been given in [6]. Due to its universal nature, it was conjectured by many that this nullset has a high Borel rank (the question is explicitely mentioned in [3] and [16]). In this paper, we refute this conjecture and show that this nullset is merely $\boldsymbol{\Sigma}_{\mathbf{3}}^{\mathbf{0}}$. Together with a result of Liang Yu, our result also implies that the exact Borel complexity of this set is $\boldsymbol{\Sigma}_{\mathbf{3}}^{\mathbf{0}}$.

To do this proof, we develop the machinery of effective randomness and effective Solovay genericity, investigating the connections between those notions and effective domination properties.
\end{abstract}

1998 ACM Subject Classification F.4.1 Mathematical Logic

Keywords and phrases Effective descriptive set theory, Higher computability, Effective randomness, Genericity

Digital Object Identifier 10.4230/LIPIcs.STACS.2014.566

\section{Introduction}

We will study in this paper the notion of forcing with closed sets of positive measure and several variants of it. This forcing is generally attributed to Solovay, who used it in [15] to produce a model of $Z F+D C$ in which all sets of reals are Lebesgue measurable. Stronger and stronger genericity for this forcing coincides with stronger and stronger notions of randomness. It is actually possible to express most of the randomness definitions that have been made over the years by forcing over closed sets of positive measure.

In the first section we give a brief overview of the part of algorithmic randomness that we need in the paper. In the second section we make a modification to the usual definition of effective Solovay genericity directly inspired by a notion introduced by Jockusch in [7] about effective genericity for Cohen forcing. This new definition will reveal itself to be interesting for its connections with effective domination properties. In the third section we will give a quick description of what we need of higher computability theory and higher randomness to approach the last section. Finally in the last section we give higher analogues of the Solovay genericity notions studied in section two, and we show again their connections with randomness and higher effective domination properties. This will allow us to conclude with the Borel complexity of the largest $\Pi_{1}^{1}$ nullset.

\section{General Background}

In this paper, we will work in the space of infinite sequences of 0's and 1's, called the Cantor space, denoted by $2^{\omega}$. We will call strings finite sequences of 0 's and 1 's, sequences elements of the Cantor space and sets the sets of sequences. For a string $\sigma$, we will denote the set of sequences extending $\sigma$ by $[\sigma]$.

The set of integers $W_{e}$ will denote the domain of the computable function $\Phi_{e}$, and $\left[W_{e}\right]$ will denote $\bigcup_{\sigma \in W_{e}}[\sigma]$, where $W_{e}$ is seen as a set of strings. We will denote by $\langle$,$\rangle a fixed$

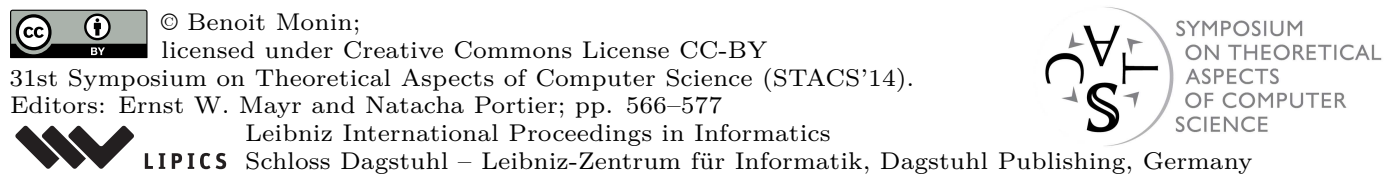


computable pairing function from $\omega \times \omega$ to $\omega$.

We will consider computable functionals (computable functions using sequences as oracles) as functions from the Cantor space to the Baire space. Then a computable functional $\Phi$ is considered define on $X \in 2^{\omega}$ if $\forall n \Phi^{X}(n) \downarrow$ and we denote by $\operatorname{dom} \Phi$ the set $\left\{X \mid \forall n \Phi^{X}(n) \downarrow\right\}$. We say that a function $f$ is computable relative to $X$ or $X$-computable if there is a computable functional defined on $X$ such that $\Phi^{X}=f$.

The topology on Cantor space is generated by the basic intervals $[\sigma]=\left\{X \in 2^{\omega} \mid X \succ \sigma\right\}$ for $\sigma$ a string. For $A \subseteq 2^{\omega}$ Lebesgue-measurable, $\lambda(A)$ will denote the Lebesgue measure of $A$, which is the unique Borel measure such that $\lambda([\sigma])=2^{-|\sigma|}$ for all strings $\sigma$.

\subsection{About the arithmetical complexity of sets}

In the Cantor space, open sets can be described as countable unions of strings. We call an open set effective if it can be described as the union of a computably enumerable set of strings, i.e. if it is equal to $\left[W_{e}\right]$ for some $e$. Such a set is said to be $\Sigma_{1}^{0}$. On the other hand, when it is open but not necessarily effectively open, the set is said to be $\boldsymbol{\Sigma}_{1}^{0}$. However, a non-effective open set is always effective relatively to some oracle. If $X$ is such an oracle, we say that the set is $\Sigma_{1}^{0}(X)$. A closed set is called effective if its complement is an effective open set, in which case we say that the closed set is a $\Pi_{1}^{0}$ set. We can then continue to describe the effective Borel sets through the arithmetical hierarchy as effective unions of effective Borel set of lower complexity and as their complements. So a $\Sigma_{n+1}^{0}$ set will be an effective union of $\Pi_{n}^{0}$ sets, and a $\Pi_{n+1}^{0}$ set will be the complement of a $\Sigma_{n+1}^{0}$ set. For example, a set $A$ is $\Sigma_{4}^{0}$ if we have an integer $e$ such that $A=\bigcup_{m_{1} \in W_{e}} \bigcap_{m_{2} \in W_{m_{1}}} \bigcup_{m_{3} \in W_{m_{2}}}\left[W_{m_{3}}\right]^{c}$.

We have a canonical surjection from integers to $\Sigma_{1}^{0}$ sets (The one which associates to $e$ the computably enumerable set $\left.\left[W_{e}\right]\right)$, but also from integers to $\Sigma_{n}^{0}$ sets for a fixed $n$. In the above example, with $n=4$ the integer $e$ is associated to the $\Sigma_{4}^{0}$ set $A$. In this context $e$ will be called an index for the set $A$.

Also for a computably enumerable set of integers $W$, we denote by $W[t]$ the enumeration of $W$ up to stage $t$. We extend this definition to effective open sets: if $O=[W]$, then $O[t]=[W[t]]$. Similarly, if $F=O^{c}, F[t]=O[t]^{c}$.

\subsection{About algorithmic randomness}

In 1966, Martin-Löf gave in [10] a definition capturing elements of the Cantor space that can be considered 'random'. Many nice properties of the Martin-Löf random sequences make this notion of randomness one of the most interesting and one of the most studied.

Intuitively a random sequence should not have any atypical property. A property is here considered atypical if the set of sequences having it is of measure 0 . It also makes sense to consider only properties which can be described in some effective way (because any $X$ has the property of being in the set $\{X\}$ and thus nothing would be random).

Definition 1. An intersection of measurable sets $\bigcap_{n} A_{n}$ is said to be effectively of measure 0 if the function which to $n$ associates the measure of $A_{n}$ is bounded by a decreasing computable function whose limit is 0 . A Martin-Löf test is a $\Pi_{2}^{0}$ set $\bigcap_{n} O_{n}$ effectively of measure 0 . We say that $X \in 2^{\omega}$ is Martin-Löf random if it is in no Martin-Löf test.

One can iterate this idea by considering $\Pi_{n}^{0}$ sets effectively of measure 0 for any $n \geq 2$. Martin-Löf randomness is also called 1-randomness, the use of $\Pi_{3}^{0}$ sets effectively of 
measure 0 gives us 2-randomness, $\Pi_{4}^{0}$ sets give us $\mathbf{3}$-randomness, and so on. The requirement for a Martin-Löf test to be effectively of measure 0 is important and leads to very nice properties. In particular there exists a universal Martin-Löf test, i.e. a test containing all the others (see [10]). This is not the case anymore if we drop the 'effectively of measure 0' condition. Instead we get a notion known as weak-2-randomness.

- Definition 2. We say that $X \in 2^{\omega}$ is weakly-2-random if it is in no $\Pi_{2}^{0}$ nullset.

As a randomness notion, weak-2-randomness is a strictly stronger than 1-randomness, but is strictly weaker than 2-randomness (see [13] section 3.6).

\section{Solovay genericity and its variants}

Cohen introduced in [4] the general technique of forcing by forcing with all dense open sets of the Cantor space (with the usual topology) in a countable model of ZFC. The most basic effective version of this would be to say that $X$ is generic if it belongs to all dense $\Sigma_{1}^{0}$ sets, a notion introduced by Kurtz in [9]. Jockusch introduced and studied in [7] a slightly different notion.

- Definition 3 (Kurtz, Jockusch). We say that $X$ is weakly-1-generic if it belongs to all dense $\Sigma_{1}^{0}$ sets. We say that $X$ is 1 -generic if for any $\Sigma_{1}^{0}$ set $U$, either $X$ belongs to $U$ or $X$ belongs to some other $\Sigma_{1}^{0}$ set $U^{\prime}$ disjoint from $U$.

We will apply Jockusch's idea behind 1-genericity to forcing with $\Pi_{1}^{0}$ sets. First note that by definition, the weakly-2-randoms are exactly the sequences which are in all $\Sigma_{2}^{0}$ sets of measure 1. If we consider the topology generated by $\Pi_{1}^{0}$ sets of positive measure, because $\Sigma_{2}^{0}$ sets of measure 1 are then dense open sets for this topology, we also get in some sense a genericity notion.

\subsection{Forcing with $\Pi_{1}^{0}$ sets}

Adding a measure requirement to the definition of genericity will always link us to randomness. We study what happens if we drop the measure requirement and if we consider instead the $\Sigma_{2}^{0}$ sets which are dense for the topology generated by the $\Pi_{1}^{0}$ sets, i.e. the $\Sigma_{2}^{0}$ sets which intersect all non-empty $\Pi_{1}^{0}$ set. It is clear that the Cantor space with this topology is a Baire space, i.e. has the property that an intersection of dense open sets is dense. This directly comes from the fact that a decreasing intersection of non-empty closed sets is non-empty. This justifies the following definition:

- Definition 4. Let $\left\{G_{i}\right\}_{i \in \omega}$ be the collection of all $\Sigma_{2}^{0}$ sets which intersect all the $\Pi_{1}^{0}$ sets. We say that $X$ is weakly- $\Pi_{1}^{0}$-generic if it belongs to $\bigcap_{i} G_{i}$.

As the next proposition shows, weak- $\Pi_{1}^{0}$-genericity has nothing to do with randomness.

- Proposition 5. No weakly- $\Pi_{1}^{0}$-generic sequence is 2-random.

Proof. We construct uniformly in $n$ a $\Sigma_{2}^{0}$ set intersecting all $\Pi_{1}^{0}$ sets and with measure smaller than $2^{-n}$. Let $\left\{F_{e}\right\}_{e \in \omega}$ be an enumeration of the $\Pi_{1}^{0}$ sets. For each $e$ we initialize $\sigma_{e}$ to the first string (using lexicographic order) of length $n+e+1$. Our $\Sigma_{2}^{0}$ set will consist of a computably enumerable set $A$ of indices of $\Pi_{1}^{0}$ sets. We now describe the algorithm to enumerate elements of $A$ : At stage $t$, for each substage $e<t$ in increasing order, if the index of $F_{e} \cap\left[\sigma_{e}\right]$ has not been enumerated yet into $A$, then enumerate it. After that, 
if $\left(F_{e} \cap\left[\sigma_{e}\right]\right)[t]=\emptyset$ then reset $\sigma_{e}$ to be the string of length $n+e+1$ following $\sigma_{e}$ in the lexicographic order. If $\sigma_{e}$ is already the last such string, leave it unchanged.

Let us prove that the measure of the $\Sigma_{2}^{0}$ set represented by $A$ is smaller than $2^{-n}$. For each $e$, if $F_{e} \cap\left[\sigma_{e}\right]=\emptyset$ then by compactness $\left(F_{e} \cap\left[\sigma_{e}\right]\right)[t]=\emptyset$ for some $t$. Thus at most one string $\sigma_{e}$ of length $n+e+1$ such that $F_{e} \cap\left[\sigma_{e}\right] \neq \emptyset$ has been enumerated into $A$, and the measure of $A$ is bounded by $\sum_{e} 2^{-n-e-1} \leq 2^{-n}$. Now our $\Sigma_{2}^{0}$ set is dense because if $F_{e}$ is not empty then there exists a string $\sigma_{e}$ of length $n+e+1$ such that $F_{e} \cap\left[\sigma_{e}\right]$ is not empty and then $A$ will intersect $F_{e}$.

From this we can then construct a $\Pi_{3}^{0}$ set effectively of measure 0 and containing all the weakly- $\Pi_{1}^{0}$-generic sequences.

Following Jockusch's 1-genericity idea we now define $\Pi_{1}^{0}$-genericity:

Definition 6. A sequence $X$ is $\Pi_{1}^{0}$-generic if for all $\Sigma_{2}^{0}$ sets $G$, either $X$ is in $G$ or there is a $\Pi_{1}^{0}$ set $F$ disjoint from $G$ such that $X$ is in $F$.

We now establish a simple but surprising connection with computability theory, which appears to be previously unknown. We say that a sequence $X$ is computably dominated if for every total function $f: \omega \rightarrow \omega$, computable relative to $X$, there exists a total computable function $g$ such that $g$ dominates $f$ (i.e. $\forall n f(n) \leq g(n)$ ).

- Proposition 7. A set $X$ is $\Pi_{1}^{0}$-generic iff it is computably dominated.

Proof. Suppose $X$ is computably dominated and take any $\Sigma_{2}^{0}$ set $\bigcup_{n} F_{n}$. Suppose that $X$ belongs to its complement, a $\Pi_{2}^{0}$ set $\bigcap_{n} O_{n}$. Let us define the $X$-computable function $f: \omega \rightarrow \omega$ which to $n$ associates the smallest $t$ so that $X \in O_{n}[t]$. As $X$ is computably dominated, there is a computable function $g$ which dominates $f$. Then $X \in \bigcap_{n} O_{n}[g(n)]$, an effectively closed set disjoint from $\bigcup_{n} F_{n}$.

Conversely suppose that $X$ is $\Pi_{1}^{0}$-generic and consider a functional $\Phi$, defined on $X$. We have that $\operatorname{dom} \Phi=\left\{X \mid \forall n \Phi^{X}(n) \downarrow\right\}$ is a $\Pi_{2}^{0}$ set containing $X$. But then as $X$ is $\Pi_{1}^{0}$-generic, it is contained in a $\Pi_{1}^{0}$ set $F$ contained in the domain of $\Phi$. Let us now build ${ }^{1}$ a computable function $f$ such that $\forall X \in F \Phi^{X}<f$. To compute the value of $f(n)$ we find the smallest pair $\langle m, t\rangle$ such that for all strings $\sigma$ of size $m$ with $[\sigma] \subseteq F[t]$, the functional $\Phi$ halts on $n$ in less than $t$ steps with $\sigma$ as an oracle (considering that if $\Phi$ needs to use bits of the oracle at positions bigger than $|\sigma|$, it does not halt). Then we set $f(n)$ to the sum of all those values plus one. All we need to show is that $f$ is total. Fix $n$ and let us prove there is a $m$ so that for all $X \in F$ we have $\Phi^{X \uparrow_{m}}(n) \downarrow$. Suppose not, then for all $m$ there is $X \in F$ with $\Phi^{\sigma_{m}}(n) \uparrow$ where $\sigma_{m}=X \uparrow_{m}$. As $\left\{\sigma_{m}\right\}_{m \in \omega}$ is infinite it has at least one limit sequence $Y$ and as $F$ is closed we have $Y \in F$. Also as $\Phi^{Y{ }^{\top} m}(n) \uparrow$ for all $m$ we have that $\Phi$ is not defined on $Y$ which contradicts the hypothesis. Thus for some $t$ we have that $F[t]$ is covered by a finite union $\bigcup_{i<k}\left[\sigma_{i}\right]$ such that $\Phi^{\sigma_{i}}(n) \downarrow$. It follows that for some $t$ and some $m$ we have that $\Phi^{\sigma}(n)$ halts in less than $t$ steps for all strings $\sigma$ of size $m$ such that $[\sigma] \subseteq F[t]$.

A direct computation shows that the set of computably dominated sequences is $\Pi_{4}^{0}$. The above proposition lowers down the Borel complexity to $\boldsymbol{\Pi}_{3}^{\mathbf{0}}$ : if for every set $A$ we denote by $A^{\circ}$ the interior of $A$ for the topology generated by $\Pi_{1}^{0}$ sets, i.e. the union of all $\Pi_{1}^{0}$ sets included in $A$, then the set of computably dominated sequences is the intersection over all

\footnotetext{
1 One can also directly deduce the existence of such a function $f$ using the fact that the supremum of a
} computable function, over an effectively compact set, is right-ce. 
the $\Pi_{2}^{0}$ sets $P$, of $P^{\circ} \cup P^{c}$. We now give a lower bound on the Borel complexity of the computably dominated sequences, however we do not know if it can be $\boldsymbol{\Sigma}_{\mathbf{3}}^{\mathbf{0}}$.

- Proposition 8. The set of computably dominated sequences is neither $\boldsymbol{\Sigma}_{\mathbf{2}}^{\mathbf{0}}$ nor $\boldsymbol{\Pi}_{\mathbf{2}}^{\mathbf{0}}$.

Proof. Let us show that it is not $\boldsymbol{\Pi}_{2}^{0}$. First note that for any $\mathbf{\Pi}_{2}^{0}$ set $A$, if $A$ is dense (for the usual topology) in some $[\sigma]$ then it contains a weakly-1-generic sequence as defined by Kurtz. Indeed, the intersection of $A \cap[\sigma]$ with all dense $\Sigma_{1}^{0}$ sets will not be empty and will then contain weakly-1-generic sequences. But by a result of computability theory (see [9]), no weakly-1-generic is computably dominated. Thus a $\boldsymbol{\Pi}_{2}^{0}$ set containing only computably dominated sequences is nowhere dense. But as the set of computably dominated sequences is dense, being closed under finite change of prefixes, such a $\boldsymbol{\Pi}_{2}^{0}$ set cannot contain all of them.

To show that it is not $\boldsymbol{\Sigma}_{\mathbf{2}}^{0}$, we adapt a technique that Liang Yu exposed in [16]. Suppose that the set of computably dominated sequences is described as $\bigcup_{n} F_{n}$ with each $F_{n}$ closed. For each $n$ let $B_{n}=\bigcup\left\{T \mid T \cap F_{n}=\emptyset\right.$ and $T$ is a $\Pi_{1}^{0}$ set with no computable member $\}$. Let us prove that the set $B_{n}$ intersects any non-empty $\Pi_{1}^{0}$ set with no computable members. Take any non-empty $\Pi_{1}^{0}$ set $G$ with no computable members. By a classical result of computability theory (see [13] proposition 1.5.12 combined with fact 1.8.36) $G$ contains a non-computably dominated sequence. Thus $G$ contains a sequence $X$ which is not in $F_{n}$. Then as $F_{n}$ is closed there is a string $\sigma$ such that $X \in G \cap[\sigma]$ but $G \cap[\sigma] \cap F_{n}=\emptyset$. Thus $G \cap[\sigma]$ is a non-empty $\Pi_{1}^{0}$ set with no computable sequence, intersecting $G$ and disjoint from $F_{n}$. Consequently we have $B_{n} \cap G \neq \emptyset$ and then each $B_{n}$ is dense for the topology generated by $\Pi_{1}^{0}$ sets with no computable member. It follows that $\bigcap_{n} B_{n}$ is also dense for this topology. From Proposition 7 the set of computably dominated sequences is also dense for this topology. Then there is a computably dominated sequence in $\bigcap_{n} B_{n}$. But we also have by design of the $B_{n}$ that $\bigcap_{n} B_{n} \cap \bigcup_{n} F_{n}=\emptyset$, which contradicts the fact that $\bigcup_{n} F_{n}$ contains all computably dominated sequences.

\subsection{Forcing with $\Pi_{1}^{0}$ sets of positive measure}

We now introduce a notion of genericity which is a measure-theoretic variation of $\Pi_{1}^{0}$-genericity defined in the previous section. The notion will be interesting for its counterpart in Higher computability. Let us now come back to the topology generated by $\Pi_{1}^{0}$ sets of positive measure. To obtain weak-2-randomness we consider only $\Sigma_{2}^{0}$ sets of measure 1 . We now consider all $\Sigma_{2}^{0}$ sets which intersect with positive measure every $\Pi_{1}^{0}$ set of positive measure.

- Definition 9. Let $\left\{G_{i}\right\}_{i \in \omega}$ be the collection of all $\Sigma_{2}^{0}$ sets $A$ such that for any $\Pi_{1}^{0}$ set $F$ of positive measure we have $\lambda(A \cap F)>0$. Then we say that $X$ is weakly- $\Pi_{1}^{0}$-Solovay-generic if it belongs to $\bigcap_{i} G_{i}$.

- Definition 10. We say that $X$ is $\Pi_{1}^{0}$-Solovay-generic if for any $\Sigma_{2}^{0}$ set $A$, either $X$ is in it or there exists a $\Pi_{1}^{0}$ set $F$ of positive measure and disjoint from $A$ such that $X$ is in it.

- Proposition 11. A set $X$ is $\Pi_{1}^{0}$-Solovay-generic iff it is weakly-2-random and computably dominated.

Proof. Suppose that $X$ is weakly-2-random and computably dominated. Take any $\Sigma_{2}^{0}$ set and suppose that $X$ does not belong to it. By Proposition 7 , as $X$ is computably dominated, we have that $X$ belongs to some $\Pi_{1}^{0}$ set disjoint from the $\Sigma_{2}^{0}$ set. Also as $X$ is weakly-2-random this $\Pi_{1}^{0}$ set has positive measure.

Conversely, suppose that $X$ is $\Pi_{1}^{0}$-Solovay-generic. In particular it is weakly-2-random and $\Pi_{1}^{0}$-generic. Then by Proposition 7 we have that it is computably dominated. 


\subsection{A separation for weak and non weak-genericity}

We will now prove that weak-genericity is not enough to obtain computable domination. For this we shall adapt a proof of a theorem in [1] saying that for any function $f$, there is a weakly-2-random $X$ and an $X$-computable function $g$ not dominated by $f$. Here we want weak- $\Pi_{1}^{0}$-Solovay-genericity instead of weak-2-randomness.

- Proposition 12. For any function $f: \omega \rightarrow \omega$ there is an $X$ weakly- $\Pi_{1}^{0}$-Solovay-generic computing a function $g: \omega \rightarrow \omega$ which is above $f$ infinitely often.

The reader can see [12] for a proof of proposition 12, that we skip here, due to its length. Using Proposition 12, we have some weakly- $\Pi_{1}^{0}$-Solovay-generics which are not computably dominated and so not $\Pi_{1}^{0}$-Solovay-generic. One can prove that weakly- $\Pi_{1}^{0}$-Solovay-genericity implies weakly- $\Pi_{1}^{0}$-genericity by showing that any $\Sigma_{2}^{0}$ set intersecting all the $\Pi_{1}^{0}$ sets also intersects with positive measure all $\Pi_{1}^{0}$ sets of positive measure. Take any $\Sigma_{2}^{0}$ set intersecting all the $\Pi_{1}^{0}$ sets. Take now a set $F$ of positive measure and consider the $\Sigma_{2}^{0}$ set $\bigcup_{n} F_{n}$ of Martin-Löf randoms (the complement of the universal Martin-Löf test). As it has measure 1 , there is some $F_{n}$ such that $F \cap F_{n}$ has positive measure. But by hypothesis our $\Sigma_{2}^{0}$ set intersects $F \cap F_{n}$. The intersection contains only Martin-Löf random sequences and thus is necessarily of positive measure. Thus there is also some weakly- $P i_{1}^{0}$-generics which are not $\Pi_{1}^{0}$-generics.

\section{Background on higher computability and higher randomness}

We now give a few definitions of higher computability and higher randomness. The Turing reductions are replaced by hyperarithmetical reductions. One intuitive way to understand a hyperarithmetical computation is to think of a standard Turing computation, but with an infinite-time Turing machine. For those machines the computational time is not an integer anymore, but an ordinal. Tapes are infinite and pre-filled with 0's, at a successor stage everything happens as in a regular Turing machine. At a limit stage, the machine changes to a special 'limit' state, the head comes back to the first cell of the first tape and if the value of a cell of a tape does not converge, it is reset to 0 (otherwise it is set to the limit of its previous values). The rest works as usual.

For example, we can build the ordinal time Turing machine which on a tape, at finite computation time $t=\langle s, e\rangle$ write 1 on the cell number $e$ of this tape if the program number $e$ halts in less than $s$ steps. At ordinal time $\omega$ we then have the halting problem on this tape. Then stages $\omega+n$ can be used to compute what one could compute with the halting problem. This can be iterated to compute anything that could be computed in a finite jump. But we can even go beyond a finite jump and continue through the ordinal jumps. To formalize this properly we need to fix the notion of notation for computable ordinals.

\subsection{Computable ordinals}

More details about this section can be found in [14]. An ordinal is defined as the order type of a well-ordered set. When the ordinal is infinite and countable it can be the order-type of a well-ordered set with domain $\omega$. We say that a countable ordinal $\alpha$ is computable if we have a relation $R \subseteq \omega \times \omega$ which is a well-founded linear order of a subset of $\omega$ of order-type $\alpha$ and if there is some $e$ such that $(n, m) \in R \leftrightarrow\langle n, m\rangle \in W_{e}$. In this case we say that $e$ codes for $\alpha$ and we write $|e|=\alpha$. Let us denote by $\mathcal{W}$ the set of integers which code for 
computable ordinals and let us denote by $\mathcal{W}_{\alpha}$ the set of integers which code for computable ordinals strictly smaller than $\alpha$.

As there are uncountably many countable ordinals, not all of them are computable. Moreover it is known that they form a strict initial segment of the countable ordinals. We denote by $\omega_{1}^{c k}$ the smallest non-computable ordinal. This notion can then be relativised. We say that $e$ is an $X$-code for the ordinal $\alpha$ if we have a relation $R \subseteq \omega \times \omega$ which is a well-founded linear order of a subset of $\omega$ of order-type $\alpha$ and if $(n, m) \in R \leftrightarrow\langle n, m\rangle \in W_{e}^{X}$. We then write $|e|^{X}=\alpha$. We denote by $\mathcal{W}^{X}$ the set of $X$-codes for $X$-computable ordinals, and we denote by $\mathcal{W}_{\alpha}^{X}$ the set of $X$-codes for $X$-computable ordinals strictly smaller than $\alpha$. Finally, we call $\omega_{1}^{X}$ the smallest ordinal which is non-computable relatively to $X$. Note that any countable ordinal is computable with a representation of itself as an oracle.

\subsection{Second order definable sets}

We say that a sequence $X$ is hyperarithmetic if for some computable function $f$ and some computable ordinal $\alpha$ we have $n \in X \leftrightarrow f(n) \in \mathcal{W}_{\alpha}$. One can define the hyperarithmetic sequences equivalently as the sequences we can Turing-compute with sufficiently many successive effective joins and iterations of the jump, constructed by induction over the computable ordinals. Also coming back to the analogy with infinite-time Turing machines we have in [5] a theorem saying that a sequence $X$ is hyperarithmetic iff it can be computed by an infinite-time Turing-machine in a computable ordinal length of time. Similarly we define what is hyperarithmetic for sets. We say that $A \subseteq 2^{\omega}$ is hyperarithmetic if there exists $e$ and $\alpha$ computable such that $X \in A \leftrightarrow e \in \mathcal{W}_{\alpha}^{X}$.

We now define $\Pi_{1}^{1}$ sequences. While hyperarithmetic sequences can be considered to be the higher counterpart of computable sequences, $\Pi_{1}^{1}$ sequences can be considered to be the higher counterpart of computably enumerable sequences. They are the sequences one can define with a formula of arithmetic containing arbitrary many first order quantifications and only universal second order quantifications (with no negations in front of them). We have another equivalent definition. A sequence $X$ is $\Pi_{1}^{1}$ if for some computable function $f$ we have $n \in X \leftrightarrow \exists \alpha<\omega_{1}^{c k} f(n) \in \mathcal{W}_{\alpha}$. Coming back to the analogy with infinite-time Turing machines, the $\Pi_{1}^{1}$ sequences also correspond to the sets of integers one can enumerate along computable ordinal length of time with such a machine (when we interpret sequences as sets of integers, considering that $n$ in the set iff the $n$-th bit of the sequence is one). The $\Sigma_{1}^{1}$ sequences are their complements (again, when we see sequences as sets of integers), the higher equivalent of co-recursively enumerable sequences. Finally a set $A$ is $\Pi_{1}^{1}$ if we have an integer $e$ so that $X \in A \leftrightarrow \exists \alpha<\omega_{1} e \in \mathcal{W}_{\alpha}^{X}$. We also have a canonical surjection from integers to $\Pi_{1}^{1}$ sets, so like the arithmetical sets, they can be indexed (in the above example, $e$ is an index for the $\Pi_{1}^{1}$ set $A$ ).

A set is called $\Delta_{1}^{1}$ if it is both $\Sigma_{1}^{1}$ and $\Pi_{1}^{1}$. By a theorem of Kleene (see chapter 2 in [14]) they are exactly the hyperarithmetical sets. An index for a $\Delta_{1}^{1}$ set will consist of a pair of two indices. One expressing it as a $\Pi_{1}^{1}$ predicate and one expressing its complement as a $\Pi_{1}^{1}$ predicate.

Note that for $\Pi_{1}^{1}$ sets, the existential quantification over the ordinals goes up to $\omega_{1}$. Indeed, if $\omega_{1}^{X}>\omega_{1}^{c k}$ it is possible that $X \in A$ is witnessed by some $X$-code $e$ for $\alpha \geq \omega_{1}^{c k}$. This leads us to a $\Pi_{1}^{1}$ set of great importance for this paper, the set $\left\{X \mid \omega_{1}^{X}>\omega_{1}^{c k}\right\}$ (the proof that this set if $\Pi_{1}^{1}$ can be found in section 9.1 of [13]). We now state two theorems that will be useful for the rest of the paper. 
- Theorem 13 (Sacks [14]). Uniformly in $\varepsilon$ and an index for a $\Delta_{1}^{1}$ set $A$, one can compute an index for a $\Sigma_{1}^{1}$ closed set $F$ so that $F \subseteq A$ and $\lambda(A-F) \leq \varepsilon$. Also one can uniformly from an index of a $\Delta_{1}^{1}$ set obtain an index for the $\Delta_{1}^{1}$ real being the measure of this set.

- Theorem 14 (Spector [14]). If $f: \omega \rightarrow \mathcal{W}^{X}$ is a total $\Pi_{1}^{1}(X)$ functional predicate then $\sup _{n}|f(n)|<\omega_{1}^{X}$.

\subsection{Higher randomness}

We now introduce notions of randomness which are higher effective variations of the usual randomness notions.

- Definition 15 (Sacks). We say that $X \in 2^{\omega}$ is $\Delta_{1}^{1}$-random if it is in no $\Delta_{1}^{1}$ nullset.

Martin-Löf was actually the first to promote this notion (see [11]), suggesting that it was the appropriate mathematical concept of randomness. Even if his first definition undoubtedly became the most successful over the years, this other definition got a second wind recently on the initiative of Hjorth and Nies who started to study the analogy between the usual notions of randomness and their higher counterparts. In order to do so they created in [6] a higher analogue of Martin-Löf randomness.

- Definition 16 (Hjorth, Nies). A $\Pi_{1}^{1}$-Martin-Löf test is given by an effectively null intersection of open sets $\bigcap_{n} O_{n}$, each $O_{n}$ being $\Pi_{1}^{1}$ uniformly in $n$. A sequence $X$ is $\Pi_{1}^{1}$-ML-random if it is in no $\Pi_{1}^{1}$-Martin-Löf test.

This definition is strictly stronger than $\Delta_{1}^{1}$-randomness (see Corollary 9.3.5 in [13]). The higher analogue of weak-2-randomness has also been studied (see [3]).

- Definition 17. We say that $X$ is weakly- $\Pi_{1}^{1}$-random if it belongs to no $\bigcap_{n} O_{n}$ with each $O_{n}$ open set $\Pi_{1}^{1}$ uniformly in $n$ and with $\lambda\left(\bigcap_{n} O_{n}\right)=0$.

Earlier, Sacks gave an even stronger definition, made possible by a theorem of Lusin saying that even though $\Pi_{1}^{1}$ sets are not necessarily Borel, they remain all measurable.

- Definition 18 (Sacks). We say that $X \in 2^{\omega}$ is $\Pi_{1}^{1}$-random if it is in no $\Pi_{1}^{1}$ nullset.

This last definition is of great importance. Kechris proved that there is a universal $\Pi_{1}^{1}$ nullset, in the sense that it contains all the others (see [8]). Later, Hjorth and Nies gave in [6] an explicit construction of this $\Pi_{1}^{1}$ nullset. Chong and Yu proved in [3] that weakly- $\Pi_{1}^{1}$-randomness is strictly stronger than $\Pi_{1}^{1}$-Martin-Löf-randomness, but it is still unknown whether $\Pi_{1}^{1}$-randomness coincides with weakly- $\Pi_{1}^{1}$-randomness.

To separate the two notions, the idea of showing they have different Borel complexity was promoted in [3]. In the next section we show that this will not be possible, by proving that the biggest $\Pi_{1}^{1}$ nullset has the surprisingly small Borel complexity of $\boldsymbol{\Sigma}_{\mathbf{3}}^{\mathbf{0}}$. Using results of [17] we will conclude that the Borel complexity of both the weakly-2-randoms and the $\Pi_{1}^{1}$-randoms, is strictly $\Pi_{3}^{0}$. We now give some important results about higher randomness, that will be needed to achieve this:

- Theorem 19 (Sacks). The set $\left\{X \mid \omega_{1}^{X}>\omega_{1}^{c k}\right\}$ has measure 0 .

Thus no $X$ such that $\omega_{1}^{X}>\omega_{1}^{c k}$ is $\Pi_{1}^{1}$-random. The following beautiful theorem of Chong, $\mathrm{Yu}$ and Nies (see [2]) strengthens Sacks' theorem:

- Theorem 20 (Chong, Yu, Nies). A sequence $X$ is $\Pi_{1}^{1}$-random iff it is $\Delta_{1}^{1}$-random and $\omega_{1}^{X}=\omega_{1}^{c k}$. 
One could also define the randomness notion introduced by $\Sigma_{1}^{1}$ nullsets, but this turns out to be equivalent to $\Delta_{1}^{1}$-randomness.

- Theorem 21 (Sacks). A $\Delta_{1}^{1}$-random sequence is in no $\Sigma_{1}^{1}$ nullset. Therefore $\Sigma_{1}^{1}$-randomness coincides with $\Delta_{1}^{1}$-randomness.

\section{$5 \quad$ Higher Solovay genericity and its variants}

- Definition 22. We say that $X$ is weakly- $\Sigma_{1}^{1}$-Solovay-generic if it belongs to all sets of the form $\bigcup_{n} F_{n}$ which intersect with positive measure all the $\Sigma_{1}^{1}$ closed sets of positive measure, where each $F_{n}$ is a $\Sigma_{1}^{1}$ closed set uniformly in $n$.

- Definition 23. We say that $X$ is $\Sigma_{1}^{1}$-Solovay-generic if for any set of the form $\bigcup_{n} F_{n}$ where each $F_{n}$ is a $\Sigma_{1}^{1}$ closed set uniformly in $n$, either $X$ is in $\bigcup_{n} F_{n}$ or $X$ is in some $\Sigma_{1}^{1}$ closed set of positive measure $F$, disjoint from $\bigcup_{n} F_{n}$.

As in the lower case, one could drop the measure requirement in the definition of $\Sigma_{1}^{1}$ Solovay-genericity and obtain interesting relations with domination properties. However we will focus in this paper only on (weakly-) $\Sigma_{1}^{1}$-Solovay-genericity.

Unlike in the lower case, we have that the set of weakly- $\Sigma_{1}^{1}$-Solovay-generics is of measure 1. We can actually prove easily that they coincide with the weakly- $\Pi_{1}^{1}$-randoms. Let $\bigcup_{n} F_{n}$ be a uniform union of $\Sigma_{1}^{1}$ closed sets with measure strictly smaller than 1. Let $\bigcap_{n} O_{n}$ be its complement. As it is a $\Pi_{1}^{1}$ set, we have $e$ such that $X \in \bigcap_{n} O_{n} \leftrightarrow \exists \alpha<\omega_{1} \quad e \in \mathcal{W}_{\alpha}^{X}$. But by Theorem 19 we have that $\left\{X \mid \exists \alpha \geq \omega_{1}^{c k} \quad e \in \mathcal{W}_{\alpha}^{X}\right\} \subseteq \mathcal{S}$ is of measure 0 . Thus for some computable $\alpha$ we have that $\left\{X \mid e \in \mathcal{W}_{\alpha}^{X}\right\}$ has positive measure. As it is a $\Delta_{1}^{1}$ set, we can find using Theorem 13 a $\Sigma_{1}^{1}$ closed set of positive measure contained in it. Thus $\bigcup_{n} F_{n}$ does not intersect all $\Sigma_{1}^{1}$ closed sets of positive measure. Conversely a uniform union of $\Sigma_{1}^{1}$ closed sets of measure 1 intersects with positive measure any $\Sigma_{1}^{1}$ closed set of positive measure. Then the weakly- $\Sigma_{1}^{1}$-Solovay-generics are exactly the weakly- $\Pi_{1}^{1}$-randoms.

We will now prove that the notion of $\Sigma_{1}^{1}$-Solovay-genericity is exactly the notion of $\Pi_{1}^{1}$-randomness. As explained at the end of the section (after Theorem 26), one can also consider this equivalence as the higher counterpart of Proposition 11.

We already know from Theorem 20 that if $X$ is weakly- $\Pi_{1}^{1}$-random but not $\Pi_{1}^{1}$-random, then $\omega_{1}^{X}>\omega_{1}^{c k}$. We will show that if $X$ is $\Sigma_{1}^{1}$-Solovay-generic then $\omega_{1}^{X}=\omega_{1}^{c k}$ which will prove the difficult part of the equivalence.

In order to prove this, we use a technique developed by Sacks and simplified by Greenberg, to show that the set of $X$ with $\omega_{1}^{X}>\omega_{1}^{c k}$ has measure 0 . First note that if $\omega_{1}^{X}>\omega_{1}^{c k}$ then there is $o \in \mathcal{W}^{X}$ such that $|o|^{X}=\omega_{1}^{c k}$. In particular for each $n$ we can uniformly restrain the relation coded by $o$ to all elements smaller than $n$. If $|o|^{X}$ is a limit ordinal this gives a set of $X$-codes for ordinals smaller than $|o|^{X}$ but cofinal (i.e. unbounded) in $|o|^{X}$. Thus if $\omega_{1}^{X}>\omega_{1}^{c k}$, there is a function $f: \omega \rightarrow \mathcal{W}^{X}$ computable in $X$ such that $\sup _{n}|f(n)|^{X}=\omega_{1}^{c k}$. The idea is the following. Suppose that for some $X$ we have a computable function $\Phi_{e}$ such that:

$$
\forall n \exists \alpha<\omega_{1}^{c k} \Phi_{e}^{X}(n) \in \mathcal{W}_{\alpha}^{X}
$$

Suppose also that $X$ is $\Sigma_{1}^{1}$-Solovay-generic. Then we will show that the supremum of $\left|\Phi_{e}^{X}(n)\right|$ over $n \in \omega$ is strictly smaller than $\omega_{1}^{c k}$. To show this we need an approximation lemma, which can be seen as an extension of Theorem 13.

- Lemma 24. For a $\Sigma_{1}^{1}$ predicate $S(X) \leftrightarrow \forall \alpha<\omega_{1}^{c k} e \notin \mathcal{W}_{\alpha}^{X}$, uniformly in e and n one can find a $\Sigma_{1}^{1}$ closed set $F \subseteq S$ with $\lambda(S-F) \leq 2^{-n}$. 
Proof. One can equivalently write $S(X) \leftrightarrow \forall o \in \mathcal{W} \quad e \notin \mathcal{W}_{|o|}^{X}$. Let $S_{o}$ be the predicate $e \notin \mathcal{W}_{|o|}^{X}$. If $o \in \mathcal{W}$ one can uniformly in $o$ and $e$ obtain an index for the $\Delta_{1}^{1}$ predicate $S_{o}$. The $\Pi_{1}^{1}$ index for it corresponds to the property : "There exists no bijection from $|e|$ to a strict initial segment of $|o|^{X}$ ", and a $\Pi_{1}^{1}$ index for its complement is : "There exists no infinite backward sequence in $|e|$, and there exists no bijection from $|o|^{X}$ to an initial segment of $|e| . "$ Note that if $o \notin \mathcal{W}$, the index is still well defined but does not correspond to anything specific.

Then uniformly in an index for $S_{o}$ and in $n$ we can find using Theorem 13 a $\Sigma_{1}^{1}$ closed set $F_{o}$ such that $F_{o} \subseteq S_{o}$ with $\lambda\left(S_{o}-F_{o}\right) \leq 2^{-o} 2^{-n}$. Now let us define $F(X) \leftrightarrow \forall o \in \mathcal{W} X \in F_{o}$. As an intersection of closed sets, the set $F$ is closed. And as $\mathcal{W}$ is $\Pi_{1}^{1}$ and $F_{o}$ is $\Sigma_{1}^{1}$ uniformly in $o$, we have that $F$ is $\Sigma_{1}^{1}$. To conclude we also we have that:

$$
\begin{aligned}
\lambda(S-F) & =\lambda\left(\bigcup_{o \in \mathcal{W}} S-F_{o}\right) \\
& \leq \lambda\left(\bigcup_{o \in \mathcal{W}} S_{o}-F_{o}\right) \\
& \leq \sum_{o \in \mathcal{W}} \lambda\left(S_{o}-F_{o}\right) \leq 2^{-n}
\end{aligned}
$$

We can now prove the desired theorem:

- Theorem 25. If $Y$ is $\Sigma_{1}^{1}$-Solovay-generic then $\omega_{1}^{Y}=\omega_{1}^{c k}$.

Proof. Suppose that $Y$ is $\Sigma_{1}^{1}$-Solovay-generic. For any functionnal $\Phi$, consider the set

$$
P=\left\{X \mid \forall n \quad \exists \alpha<\omega_{1}^{c k} \Phi^{X}(n) \in \mathcal{W}_{\alpha}^{X}\right\}
$$

Let $P_{n}=\left\{X \mid \exists \alpha<\omega_{1}^{c k} \Phi^{X}(n) \in \mathcal{W}_{\alpha}^{X}\right\}$ and $P_{n, \alpha}=\left\{X \mid \Phi^{X}(n) \in \mathcal{W}_{\alpha}^{X}\right\}$, so $P=\bigcap_{n} P_{n}$ and $P_{n}=\bigcup_{\alpha<\omega_{1}^{c k}} P_{n, \alpha}$.

From Lemma 24 we can find uniformly in $n$ a uniform union of $\Sigma_{1}^{1}$ closed sets included in $P_{n}^{c}$ with the same measure as $P_{n}^{c}$. From this we can find a uniform union of $\Sigma_{1}^{1}$ closed sets included in $P^{c}$ with the same measure as $P^{c}$. Suppose that $Y$ is in $P$, as it is $\Sigma_{1}^{1}$-Solovaygeneric we have a $\Sigma_{1}^{1}$ closed set $F$ of positive measure containing $Y$ which is disjoint from $P^{c}$ up to a set of measure 0 , formally $\lambda\left(F \cap P^{c}\right)=0$. In particular for each $n$ we have $\lambda\left(F \cap P_{n}^{c}\right)=0$ and then $\lambda\left(F^{c} \cup P_{n}\right)=1$. Then let $f$ be the total function which to each pair $\langle n, m\rangle$ associates the smallest code $o_{n, m} \in \mathcal{W}$ such that:

$$
\lambda\left(F_{\left|o_{n, m}\right|}^{c} \cup P_{n,\left|o_{n, m}\right|}\right)>1-2^{-m}
$$

where $F_{\alpha}^{c}$ is the $\Delta_{1}^{1}$ set of strings which are witnessed to be in $F^{c}$ via an ordinal smaller than $\alpha$. Using second part of Theorem 13 one can prove that $f$ is $\Pi_{1}^{1}$. Let $\alpha^{*}=\sup _{n, m}|f(n, m)|$. By Theorem 14 we have that $\alpha^{*}<\omega_{1}^{c k}$. Then we have:

$$
\begin{aligned}
\forall n \lambda\left(F_{\alpha *}^{c} \cup \bigcup_{\alpha<\alpha^{*}} P_{\alpha, n}\right) & =1 \\
\rightarrow \quad \forall n \lambda\left(F_{\alpha^{*}} \cap \bigcap_{\alpha<\alpha^{*}} P_{\alpha, n}^{c}\right) & =0 \\
\rightarrow \quad \forall n \lambda\left(F-\bigcup_{\alpha<\alpha^{*}} P_{\alpha, n}\right) & =0 \\
\rightarrow \quad \lambda\left(F-\bigcap_{n} \bigcup_{\alpha<\alpha^{*}} P_{\alpha, n}\right) & =0
\end{aligned}
$$

As $X$ is $\Sigma_{1}^{1}$-Solovay-generic it is in particular weakly- $\Sigma_{1}^{1}$-Solovay-generic and then weakly$\Pi_{1}^{1}$-random. Thus by Theorem 21 it belongs to no $\Sigma_{1}^{1}$ set of measure 0 . Then as $F-$ $\bigcap_{n} \bigcup_{\alpha<\alpha^{*}} P_{\alpha, n}$ is a $\Sigma_{1}^{1}$ set of measure 0 we have that $X$ belongs to $\bigcap_{n} \bigcup_{\alpha<\alpha^{*}} P_{\alpha, n}$ and then $\sup _{n} \Phi^{X}(n) \leq \alpha^{*}<\omega_{1}^{c k}$. 
We can now prove the equivalence:

- Theorem 26. The set of $\Sigma_{1}^{1}$-Solovay-generics is exactly the set of $\Pi_{1}^{1}$-randoms.

Proof. Using Theorem 20 we have that the $\Sigma_{1}^{1}$-Solovay-generics are included in the $\Pi_{1}^{1}$ randoms. We just have to prove the reverse inclusion.

Suppose $Y$ is not $\Sigma_{1}^{1}$-Solovay-generic. If $\omega_{1}^{Y}>\omega_{1}^{c k}$ then $Y$ is not $\Pi_{1}^{1}$-random. Otherwise $\omega_{1}^{Y}=\omega_{1}^{c k}$ and in this case there is a sequence of $\Sigma_{1}^{1}$ closed sets $\bigcup_{n} F_{n}$ of positive measure such that $X$ is not in $\bigcup_{n} F_{n}$ and such that any $\Sigma_{1}^{1}$ closed set of positive measure which is disjoint from $\bigcup_{n} F_{n}$ does not contain $Y$. The complement of $\bigcup_{n} F_{n}$ is a $\Pi_{1}^{1}$ set $P$ containing $Y$. Let $e$ be so that $P(X) \leftrightarrow \exists \alpha<\omega_{1} e \in \mathcal{W}_{\alpha}^{X}$. As $\omega_{1}^{Y}=\omega_{1}^{c k}$ and $P(Y)$, we have that $\exists \alpha<\omega_{1}^{c k} e \in \mathcal{W}_{\alpha}^{Y}$. But then $Y$ is in a $\Delta_{1}^{1}$ set that one can approximate using Theorem 13 by an effective union of $\Sigma_{1}^{1}$ closed sets of the same measure. Thus as $X$ can be in none of them it is in a $\Pi_{1}^{1}$ set of measure 0 and then not $\Pi_{1}^{1}$-random.

The previous theorem gives an interesting corollary, making a connection with another domination property. We say that a sequence $X$ is hyp-dominated if for every total function $f: \omega \rightarrow \omega, \Delta_{1}^{1}$ relative to $X$, there exists a total $\Delta_{1}^{1}$ function $g$ such that $g$ dominates $f$ (i.e. $\forall n f(n) \leq g(n))$. Chong, Yu and Nies proved in [2] that all $\Pi_{1}^{1}$-random sequences are hyp-dominated. It follows from that and from the previous theorem that a sequence $X$ is $\Sigma_{1}^{1}$-Solovay-generic iff it is weakly-2-random and hyp-dominated. This can be seen as the higher counterpart of Proposition 11.

We have a second corollary, giving a higher bound on the Borel complexity of the $\Pi_{1}^{1}$-randoms, and then on the biggest $\Pi_{1}^{1}$ nullset.

- Corollary 27. The set of $\Pi_{1}^{1}$-randoms is $\Pi_{3}^{0}$.

The $\Pi_{3}^{0}$ set is obtained exactly the same way we obtain the $\Pi_{3}^{0}$ set of computably dominated sequences. The following result of Liang Yu (see [17]) can be used to prove that the set of $\Pi_{1}^{1}$-randoms is not $\boldsymbol{\Sigma}_{3}^{\mathbf{0}}$.

- Theorem 28 (Liang Yu). Let $\bigcap_{n} O_{n}$ be a $\Pi_{2}^{0}$ sets contaning only weakly- $\Pi_{1}^{1}$-randoms. Then the set $\left\{F \mid F\right.$ is a $\Sigma_{1}^{1}$ closed set and $\left.\bigcap_{n} O_{n} \cap F=\emptyset\right\}$ intersects with positive measure any $\Sigma_{1}^{1}$ closed sets of positive measure.

It follows that the set of weakly- $\Pi_{1}^{1}$-randoms cannot be $\Sigma_{3}^{0}$ but also that the set of $\Pi_{1}^{1}$ randoms cannot be $\boldsymbol{\Sigma}_{\mathbf{3}}^{\mathbf{0}}$. Indeed, suppose that the set of $\Pi_{1}^{1}$-randoms is equal to $\bigcup_{n} \bigcap_{m} O_{n, m}$ each $O_{n, m}$ being open. For each $n$ let $A_{n}=\left\{F \mid F\right.$ is a $\Sigma_{1}^{1}$ closed set and $\left.\bigcap_{m} O_{n, m} \cap F=\emptyset\right\}$. We have $\bigcap_{n} A_{n} \cap \bigcup_{n} \bigcap_{m} O_{n, m}=\emptyset$, and from Theorem 28 we have that $\bigcap_{n} A_{n}$ contains some Solovay- $\Sigma_{1}^{1}$-generic elements, which contradicts that $\bigcup_{n} \bigcap_{m} O_{n, m}$ contains all of them.

The question whether it is possible for $X$ to be weakly-Solovay- $\Sigma_{1}^{1}$-generic but not Solovay$\Sigma_{1}^{1}$-generic (equivalently weakly- $\Pi_{1}^{1}$-random but not $\Pi_{1}^{1}$-random) is still open. The technique that we use in the lower case to separate weak genericity from non weak genericity does not seem to work here.

Acknowledgements. I would like to thank Laurent Bienvenu, Noam Greenberg, Paul Shafer and Liang $\mathrm{Yu}$ for helpful comments and discussions. 


\section{References}

1 George Barmpalias, Rod Downey, and Keng Meng Ng. Jump inversions inside effectively closed sets and applications to randomness. Journal of Symbolic Logic, 76(2):491-518, 2011.

2 Chi Tat Chong, André Nies, and Liang Yu. Lowness of higher randomness notions. Israel Journal of Mathematics, 166:39-60, 2008.

3 Chi Tat Chong and Liang Yu. Randomness in the higher setting. under refereeing, http: //math.nju.edu.cn/ yuliang/cy1.pdf.

4 Paul J Cohen. Set theory and the continuum hypothesis. Dover Publications, 1966.

5 Joel David Hamkins and Andy Lewis. Infinite time Turing machines. Journal of Symbolic Logic, pages 567-604, 2000.

6 Greg Hjorth and André Nies. Randomness via effective descriptive set theory. Journal of the London Mathematical Society, 75(2):495-508, 2007.

7 Carl G Jockusch Jr. Simple proofs of some theorems on high degrees of unsolvability. Canadian Journal of Mathematics, 29(5):1072-1080, 1977.

8 Alexander S Kechris. The theory of countable analytical sets. Transactions of the American Mathematical Society, 202:259-297, 1975.

9 S Kurtz. Randomness and genericity in the degrees of unsolvability. phd diss., University of Illinois. Urbana, 1981.

10 Per Martin-Löf. The definition of random sequences. Information and Control, 9:602-619, 1966.

11 Per Martin-Löf. On the notion of randomness. Studies in Logic and the Foundations of Mathematics, 60:73-78, 1970.

12 Benoit Monin. Higher randomness and forcing with closed sets, http://www.liafa. univ-paris-diderot.fr/ benoitm/ressources/papers/paper_stacs_2014.pdf, 2014.

13 Andre Nies. Computability and Randomness. Oxford University Press, 2009.

14 Gerald E Sacks. Higher recursion theory. Springer, 2010.

15 Robert M Solovay. A model of set-theory in which every set of reals is Lebesgue measurable. The Annals of Mathematics, 92(1):1-56, 1970.

16 Liang Yu. Descriptive set theoretical complexity of randomness notions. Fundamenta Mathematicae, 215:219-231, 2011.

17 Liang Yu. LogicBlog2013, Higher randomness, http://dl.dropboxusercontent.com/u/ 370127/Blog/Blog2013.pdf, 2013. 